\title{
ZUR DARSTELLUNG DER TEMPORA IN AUSGEW ̈̈HLTEN GRAMMATIKEN DES DEUTSCHEN
}

\section{ABSTRACT: Presentation of tenses in the German grammars}

The meaning of past tenses is presented in various grammars in different ways. It was especially the hypothesis of the aspect-oriented meaning of Perfekt tense that has been paid attention to; its supporters claim that German Perfekt and Plusquamperfekt tenses express the meaning of completed action. The analysis of works of scholars has proved that the basic meaning of Perfekt tense is expressing the concept of anteriority. In the following part a few grammars have been presented. Their authors analysed the meaning of Perfekt tense having discussed telic and atelic verbs.

KEYwORDS: aspect-oriented meaning of Perfect tense in German; telic and atelic verbs

\section{Vorbemerkungen}

\author{
Unter Zeiten \\ Das Perfekt und das Imperfekt \\ tranken Sekt. \\ Sie stießen aufs Futurum an \\ (was man wohl gelten kann). \\ Plusquamper und Exaktfutur \\ blinzten nur. ${ }^{1}$
}

„Zeit ist zentraler Aspekt unseres Lebens und des gesamten Universums. Sie ist etwas Unentrinnbares: Wir sind in der Zeit, wir können nicht aus der Zeit heraus" (Vater 2007: 1). Bull (1968: 4) folgend kann man zwei Typen der Zeiteinteilung unterschei-

Andrzej Kątny - Uniwersytet Gdański, filak@ug.edu.pl

${ }^{1}$ Morgenstern, Christian (1974): „Unter Zeiten“. In: Ders.: Alle Galgenlieder. Frankfurt a. M.: Insel. 
den - die persönliche und die öffentliche Zeit (,,personal and public time“): „Personal time derives, then, from man's attempt to measure duration by using his own emotions as the clock" (Bull 1968: 5). Aspekt und Tempora als grammatikalisierte Kategorien sowie lexikalische Mittel dienen zum Ausdruck der Zeitreferenz; sie wird als „Bezug sprachlicher Mittel auf Zeitrelationen zwischen Ereignissen“ (Vater 2010: 349) aufgefasst. So wie die meisten Linguisten vertrete ich die Ansicht, dass Tempora hauptsächlich Zeitrelationen ausdrücken. Comrie (1985: 8) deutet Tempus als „grammaticalized expression of location in time".

Das Scherzgedicht Morgensterns (1871-1914) stellt die Gäste der Trinkrunde (des Symposiums) dar und lässt die freundliche Nachbarschaft zwischen Perfekt und Präteritum andeuten; beide prosten einander auf die eher ungewisse Zukunft zu; Plusquamperfekt und Futur II als weit entfernte Zeitgenossen (vielleicht sogar Zaungäste) können nur neidisch zuschauen.

\section{Einleitung}

In den meisten Grammatiken des Deutschen für Polnischsprecher gab es (und gibt es noch) eine z. T. falsche Erklärung zum Perfekt und Präteritum. ${ }^{2}$ Es wird nämlich behauptet, dass dem deutschen Perfekt das polnische perfektive Präteritum und dem Imperfekt - das polnische imperfektive Präteritum entspricht. Czochralski (1975) hat wohl als erster dies in Frage gestellt ${ }^{3}$ und gezeigt, dass die beiden deutschen Tempora sowohl als „,vollzogen, abgeschlossen“ als auch als „,nicht-vollzogen“ gedeutet werden können.

Diese Sachlage hängt hauptsächlich mit den Darstellungen in den Grammatiken des Deutschen zusammen - diese haben die lateinische Grammatik als Vorbild genommen und die lateinischen Bezeichnungen ins Deutsche übersetzt. Als weitere Ursache gilt wohl in vielen Fällen die Nichtbeachtung der aktionalen Merkmale der Verben (der Aktionsarten). In diesem Kontext möchte ich auf die Tempus-Auffassungen in einigen Grammatiken eingehen.

\section{Zur Aspekthypothese}

Die Tempora werden in vielen Grammatiken des Deutschen in zwei Gruppen ${ }^{4}$ eingeteilt; zur I. Gruppe gehören Präsens, Präteritum und Futur I; zur II. Gruppe -

${ }^{2}$ Dies hing (und hängt) natürlich mit den Darstellungen in den Grammatiken des Deutschen zusammen; die Bezeichnung „,vollzogen, abgeschlossen“ wird als „,dokonany, zakończony“ verstanden und automatisch mit dem perfektiven Präteritum in Verbindung gesetzt.

${ }^{3}$ Dies hat er dann in den von ihm verfassten Grammatiken des Deutschen immer berücksichtigt.

${ }^{4}$ Die Einteilung der Tempora in zwei Gruppen findet man u. a. in Erben (1963), Schmidt (1977: 219ff.), Heidolph u. a. (1981: 511), Eisenberg (1986: 115ff.), Darski (2015: 369). Die Tempora der Grup- 
Perfekt, Plusquamperfekt und Futur II. Im Folgenden wende ich mich hauptsächlich der II. Gruppe zu und möchte zuerst die Verwendung der Begriffe ,vollzogen, abgeschlossen“ bei der Beschreibung und Analyse von Perfekt und Plusquamperfekt unter die Lupe nehmen. Einige Zitate aus den deutschen Grammatiken mögen stichwortartig die Auffassungen der Vertreter der sogenannten Aspekthypothese veranschaulichen.

Geht es [...] darum, einen Vorgang als nunmehr vollendet festzustellen, so wird die Form des sog. Perfekts (<lat. perfectus vollendet, eig. perfectum tempus) gebraucht, [...] (Erben 1963: 37)

Nicht der zeitliche Wert des jeweiligen Verbums wird im Perfekt realisiert, sondern die Tatsächlichkeit des Vollzugs. (Brinkmann 1971: 339)

Weil das Perfekt einen vollzogenen Prozeß aus dem Kontinuum des Vergangenen heraushebt, hat es die Fähigkeit, nicht nur ein Einzelereignis in seiner Bedeutung hervorzuheben, sondern auch einen größeren Verlauf zusammenfassend zu überblicken. (Brinkmann 1971: 341)

Das Perfekt hat seiner Bildung entsprechend [...] Vollzugscharakter. Es stellt fest. Der Gedanke an einen Ablauf ist ausgeschlossen. Diese aktionale Bedeutungskomponente kennzeichnet alle Varianten beim Gebrauch des Perfekts. (Jung 1980: 216)

Das Perfekt bezeichnet in Äußerungen ein Geschehen, dass im Sprech- oder Schreibzeitpunkt bereits abgeschlossen ist. Der Abschluss kann sich sowohl auf die Vergangenheit wie auch auf die Zukunft beziehen. [...] Das Perfekt stellt einzelne, schon abgeschlossene Tatsachen fest. (Darski 2015: 375)

Auch bei Engel ${ }^{5}$ (1988: 450), Heidolph u. a. (1981: 514), Schmidt (1977: 225f.) und in einigen weiteren Grammatiken wird dem Perfekt das Merkmal ,vollendet, abgeschlossen“ zugeschrieben. Thieroff (1992: 161) weist darauf hin, dass auch in einigen Auflagen der Duden-Grammatik die gleiche Deutung von Perfekt zu finden sei. Wenn man diese Erklärungen und Definitionen liest, dann entsteht bei mir (und wahrscheinlich bei den meisten Muttersprachlern der slawischen Sprachen) der Eindruck, dass das deutsche Perfekt die Bedeutung des perfektiven Aspekts involviert. Dieser Eindruck wird durch die angeführten Beispiele ${ }^{6}$ noch bekräftigt, denn in der Regel treten in den Beispielsätzen telische (grenzbezogene) $)^{7}$ Verben auf:

(1) Ich habe gute Arbeiter gewonnen.

(2) Wir haben den Brief erhalten und möchten darauf Folgendes mitteilen.

(3) Gestern hat sich an der Albertbrücke ein schwerer Verkehrsunfall ereignet. Die Polizei hat festgestellt, daß er durch die Straßenglätte verursacht worden ist.

(4) Das hast du gut gemacht!

pe I werden als „Vollzug nicht angezeigt“ (Heidolph u. a. 1981), „Verlaufsstufe“ oder ,imperfektiv (nicht abgeschlossen)“ (Eisenberg 1986: 117), die der Gruppe II - als „Vollzug angezeigt“ (Heidolph u. a. 1981), „Vollzugsstufe“ oder „perfektiv (abgeschlossen)“(Eisenberg 1986: 117) bezeichnet.

${ }^{5}$ In der Neuauflage berücksichtigt Engel (2004: 235f.) den Einfluss von aktionalen Eigenschaften auf die Bedeutungen von Perfekt und Plusquamperfekt.

${ }^{6}$ Belege 1-4 stammen aus Jung (1980: 216), 5-7 aus Darski (2015: 375) und 8-9 aus Engel (1988: 450).

${ }^{7}$ Beim Zukunftsbezug von Futur II und Perfekt ist die Verwendung eines telischen Verbs (oder u. U. des telischen Kontextes) obligatorisch; bei anderen Varianten von Futur II und Perfekt sind telische oder atelische Verben möglich. 
(5) Jetzt hat er es begriffen. Es hat geschneit.

(6) Wir haben das bereits gestern besprochen.

(7) Er ist in die Schule gegangen.

(8) Schön habt ihr die Wohnung eingerichtet, fand die Mutter. (,abgeschlossen zu einem vergangenen Zeitpunkt $t^{*}$

(9) Jetzt haben wir die Wohnung vollständig eingerichtet. (,abgeschlossen im Sprechzeitpunkt ${ }^{`}$ )

Die oben erwähnten Verfasser sprechen von ,Vollzug, Vollendung oder Abschluss“; Eisenberg (1986: 117) dagegen verwendet bei der Beschreibung der Tempora den Ausdruck „Aspekt“; für die Beschreibung der Tempora führt der Verfasser die Begriffe ,perfektiv/imperfektiv“" ein:

Ein Vorgang ist imperfektiv, wenn das Zeitintervall, in dem er sich abspielt, beiderseitig offen ist [...]. Er ist perfektiv, wenn das Zeitintervall nach rechts (>hinten $<)$ abgeschlossen ist [...].

Mit den meisten Grammatiken schreiben wir dem Tempus auch die Funktion zu, die (Nicht-) Abgeschlossenheit eines Vorgangs zu signalisieren.

Die Ausführungen von Eisenberg (1986: 121; auch in der Neuauflage von 1999) sind nicht nur für Auslandsgermanisten verwirrend, denn bisher hat man vom Aspekt im Deutschen als einer verdeckten Kategorie gesprochen: „Das Deutsche hat nicht nur ein Tempussystem von Formen, sondern der Aspekt- und Zeitbezug ist ebenfalls systematisch“.

\section{1. „Abgeschlossen, vollzogen“" im aspektuellen und im temporalen Sinne}

Mit dem Begriff „vollzogen“ bei der Deutung des Perfekts hat sich u. a. Gelhaus (1974: 227) ausführlich auseinandergesetzt:

Termini wie „vollzogen“ oder „abgeschlossen“ sind nur dort sinnvoll verwendbar, wo es sich um etwas Vollziehbares, Abschließbares handelt, d. h. wo Handlungen oder Prozesse vorliegen. Zweifelsohne ist damit ausgesagt, daß der Vorgang oder die Handlung einen Endpunkt erreicht haben, sei es einen natürlichen, sei es einen gesetzten. Eine Handlung oder einen Vorgang im Hinblick auf den Endpunkt sehen, heißt aber die Sache quantifiziert sehen, d. h. unter dem Blickpunkt einer Strecke mit Anfang oder Ende (sofern die Strecke nicht eine Null-Strecke, d. h. ein Punkt ist).

Gelhaus (1974: 228) schlussfolgert,

daß die Perfektform allein nicht „,+vollzogen“ (im Hinblick auf einen Bezugspunkt) signalisiert, sondern nur in Verbindung mit einem anderen Signal, das , +E (der Handlung oder des Vorgangs)“ angibt, d. h. daß die Perfektform qua Form nur anzeigt, daß etwa (das näher zu bestimmen ist) vor einem bestimmten Bezugspunkt BP liegt.

[...] soll durch die Perfektform „Vollzug gegeben“ (im Hinblick auf einen Bezugspunkt BP) signalisiert werden, dann kann dies nur geschehen, wenn außerhalb der Perfektform implizit oder explizit ein Terminativsignal gesetzt ist. (Gelhaus 1974: 229) 
Die hier diskutierte Fragestellung wurde auch von Andersson (1978: 100) genau untersucht; er weist darauf hin, dass man zwischen der aspektuellen und temporalen Abgeschlossenheit unterscheiden muss: „Nur das Merkmal temporale Abgeschlossenheit ist der Reihe II inhärent sowohl bei gbz [grenzbezogener] wie bei ngbz [nicht-grenzbezogener] VP. Bei gbz VP wird außerdem das Merkmal aspektuelle Abgeschlossenheit realisiert" (Andersson 1978: 100). Andersson beruft sich hier auf die Studien von Žerebkov und stellt eindeutig fest: „die Reihe II drückt aus, dass der Handlungsinhalt lokalisiert ist als früher liegend im Verhältnis zu einem anderen Zeitpunkt, auf den der Zeitvektor der finiten Konstituente (= des Hilfsverbs) bezogen ist" (Andersson 1978: 101).

Ehrich und Vater (1989: 104ff.) setzten sich mit der Frage auseinander, „ob das Perfekt ein Tempus oder ein Aspekt" ist und konnten in der Fachliteratur vier Hypothesen ausfindig machen: die Tempus-, die Ambiguitäts-, die Komplexitäts- und die Aspekthypothese; die Letztere wird u. a. in den Grammatiken von Brinkmann und Glinz vertreten. Treffend äußern sich Ehrich und Vater (1989: 105) zu der Aspekthypothese:

Gemäß dieser Hypothese ist die temporale Deutung des Perfekts als Ausdruck der Vergangenheit nur ein Epiphänomen seiner aspektuellen Bedeutung: was zu einem bestimmten Zeitpunkt t „,vollzogen“ oder „abgeschlossen“ ist, dessen „Verlauf“ liegt relativ zu t in der Vergangenheit. Perfekt und Präteritum sind nicht äquivalent: Perfekt ist ein Aspekt, Präteritum ist ein Tempus mit der Bedeutung „Vergangenheit“.

In einem neueren Beitrag von Andersson (2004: 7) kommt dies noch deutlicher zum Ausdruck:

Im aspektuellen Sinn 'abgeschlossen, vollzogen' ist ein Sachverhalt, der als zusammengesetzte Totalität gesehen wird, was bei transformativen Verben und Verbphrasen bedeutet, dass sich die Entwicklungsdynamik der Handlung erschöpft und der Nachzustand tatsächlich erreicht wird oder ist. Das Perfektsystem als Ganzes muss als [+ Anteriorität] gekennzeichnet werden, also als ein Subsystem der Temporalität und nicht als ein Subsystem der Aspektualität.

Im Weiteren stellt der Verfasser fest, dass die aspektuelle Abgeschlossenheit bei transformativen Verben (Phrasen) im passenden Kontext vermittelt werden kann; in dem von Andersson (Andersson 2004: 107) angeführten Satz (vgl. 10) wird gezeigt, dass der Kontext (die Konjunktion) trotz eines transformativen Verbs die temporale Deutung auferlegt:

(10) Während sie das Haus abgerissen haben / hatten, haben / hatten sie die ganze Zeit auf Spuren der ursprünglichen Bauweise geachtet.

Noch eine Bemerkung zum Perfekt in der eher sporadisch vorkommenden Bedeutungsvariante ,abgeschlossene, resultative Zukunft“ ist hier fällig. In diesem Falle kann man das Merkmal „,vollzogen“ durchaus gelten lassen, zumal hier nur telische, grenzbezogene Verben auftreten können. 
Dem Plusquamperfekt wird ebenfalls nur der Vollzugscharakter (,vollendet") in der Vergangenheit oder Vorvergangenheit zugewiesen - so u. a. bei $\operatorname{Erben}^{8}$ (1963: 41), Jung (1980: 217) - „Es stellt Vorgänge dar, die in der Vergangenheit bereits vollendet sind“, Brinkmann, Engel (1988: 451), Heidolph u. a. (1981) sowie Darski - „vergangen und abgeschlossen“" (2015: 375).

\subsection{Kurze Zusammenfassung}

Resümierend kann man sagen, dass es nur zum Teil richtig ist, dem Perfekt bzw. Plusquamperfekt das Merkmal ,abgeschlossen, vollendet“ zuzuschreiben - nämlich nur dann, wenn telische Verben (oder telisch interpretierbare Phrasen) im passenden Kontext in den jeweiligen Sätzen, Äußerungen vorkommen.

Das Festhalten von manchen Forschern an der sogenannten Aspekthypothese dürfte m. E. durch folgende Tatsachen erklärt werden:

- Da in bestimmten Textsorten der Gebrauch telischer Verben beim Perfekt deutlich überwiegt, könnte bei einigen Forschern der Eindruck entstanden sein, dass alle Verben sich so verhalten.

- Historische Gründe: Das deutsche Perfekt mit haben ist aus dem Resultativum entstanden, das nur von telischen, transitiven Verben gebildet werden konnte und die Abgeschlossenheit, Vollzogenheit (und die Nachphase, den Nachzustand) ausdrückte. Im Prozess der Grammatikalisierung hat das haben-Perfekt seine resultative (aspektuelle) Bedeutung verloren und wurde zum Vergangenheitstempus.

\section{Zu einigen Grammatiken, die in der Tempusbeschreibung die Aktionsarten berücksichtigen}

\subsection{Deutsche Grammatik von Helbig/Buscha}

Die Grammatik von Helbig/Buscha $(1972 ; 2001)$ ist für den Ausländerunterricht bestimmt und beschreibt einige Bereiche ausführlicher als dies in den bisher zugänglichen Grammatiken der Fall war. „Die Grammatik für den Muttersprachenunterricht kann von der Kompetenz ausgehen, eine Grammatik für den Fremdsprachenunterricht dient (als ein neben anderen auf Kenntnisvermittlung gerichtetes Mittel) dazu, diese Kompetenz aufzubauen“"(Helbig/Buscha 2001: 17). Bei der Beschreibung von Tempusformen und -bedeutungen wird auf die von Reichenbach und später von Baumgärtner/

\footnotetext{
${ }^{8}$ In einigen Beispielsätzen werden jedoch atelische Verben verwendet, z. B.: Wir saßen einander gegenüber an einem sehr hübschen alten Spieltisch ... ich hatte an glücklicheren Tagen an diesem Tische Schach gespielt.
} 
Wunderlich (1969) modifizierte Fassung der drei Parameter zurückgegriffen: Aktzeit (E: point of event), Sprechzeit (S: point od speech) und Betrachtzeit (R: point of reference). Außerdem werden die aktionalen Merkmale (als Resultativität bezeichnet), der Modalfaktor, das Vorkommen von Adverbialien sowie der Verwendungsbereich (Umgangs-, Hochsprache: Colloquial) in der Bedeutungsbeschreibung berücksichtigt. Den Beispielen kann man leicht entnehmen, dass die Differenzierung der Varianten beim Perfekt auf die aktionale Charakteristik des Verbs zurückzuführen ist (Helbig/ Buscha 2001: 135f.):

(11) Wir haben (gestern) die Stadt besichtigt. Sie sind (neulich) im Gebirge viel gewandert.

(12) Peter ist (vor einigen Stunden) eingeschlafen. (> Peter schläft jetzt)

(13) Bis zum nächsten Jahr hat er seine Dissertation abgeschlossen. ${ }^{9}$

Das Beispiel (11) wurde der Bedeutungsvariante „1. Perfekt zur Bezeichnung eines vergangenen Geschehens“, Beispiel (12) - „2. Perfekt zur Bezeichnung eines vergangenen Geschehens mit resultativem Charakter" und das Beispiel (13) der Variante „3. Perfekt zur Bezeichnung eines zukünftigen Geschehens“ zugeordnet. Der aufmerksame Beobachter kann feststellen, dass in der 1. Variante atelische und in der 2. Variante telische Verben ${ }^{10}$ vorkommen. Dies wird in den Anmerkungen unter der 2. Variante deutlich gemacht: „Diese Bedeutungsvariante ist auf transformative Verben beschränkt, da nur diese den Übergang zu einem Folgezustand bezeichnen“ (Helbig/ Buscha 2001: 136). Bei der 3. Variante müsste m. E. diese Einschränkung ${ }^{11}$ auch genannt werden, denn im Prinzip kommen hier nur telische Verben/Verbphrasen in Frage.

Beim Plusquamperfekt unterscheiden die Verfasser Plusquamperf. (1) zur Bezeichnung eines vorvergangenen Geschehens und (2) eines resultativen Geschehens in der Vergangenheit.

${ }^{9}$ Diesen Satz kann man ins Polnische mit dem perfektiven Futur übersetzen: Do przyszłego roku ukończy dysertację. oder mit Do przyszłego roku będzie miat/mieć ukończona dysertację. Die letztere Konstruktion ist für das gesprochene Polnisch charakteristisch; sie besteht aus dem Zeithilfsverb będzie und mieć [haben] + Passivpartizip eines perfektiven Verbs. Ich bezeichne sie als mieć-Zustandsform oder als (Possessiv-)Resultativ (vgl. Kątny 2010; 2011): Dysertację mam ukończona; spodnie mam uprasowane. In diesen Konstruktionen besteht Kongruenz zwischen dem Akkusativobjekt und dem Passivpartizip eines perfektiven Verbs. In einigen Arbeiten wird die Meinung vertreten, dass es sich bei diesen Konstruktionen um ein neues Perfekt im Polnischen handelt (Piskorz 2012; Abraham u. a. 2011). Im Deutschen kommt ziemlich selten eine vergleichbare Konstruktion vor: Der kleine Junge hat die Haare kurz geschnitten [Chłopiec ma włosy krótko uczesane] (Darski 2015: 242). Das in diesen Konstruktionen vorkommende Partizip II wird von Darski (2015: 242) als qualitatives Adverb bezeichnet.

${ }^{10}$ Bei der Übersetzung der Sätze der 1. Variante wird im Polnischen das imperfektive Präteritum (zwiedzaliśmy) und der Verben der 2. Variante - das perfektive Präteritum verwendet.

${ }^{11}$ Die explizite Nennung dieser Einschränkung ist notwendig, denn Germanistikstudenten verwenden hier auch atelische Verben, was natürlich falsch ist. Die gleiche Beschränkung betrifft auch Futur II in der Variante abgeschlossene (oder resultative) Zukunft. Die einzige mir bekannte Grammatik, die dies hervorhebt, ist die von Žerebkov (1975: 103, 106). 


\subsection{Die Deutsch-polnische kontrastive Grammatik}

Die Deutsch-polnische kontrastive Grammatik (=DpG) baut auf der Dependenz-Verb-Grammatik von Engel (1988) auf; im Zusammenhang damit sind das Modell sowie auch viele Einzellösungen ${ }^{12}$ übernommen worden - u. a. die Einteilung in finite Verben und temporale Verbalkomplexe, der Verzicht auf ein Tempussystem, die Anrechnung von werden zu den Modalverben u. a. Als ein Novum ist die Berücksichtigung der aktionalen Bedeutung des Verbs bei der Analyse von Perfekt einzuschätzen:

Das Perfekt bedeutet somit bei telischen Verben, dass ein Sachverhalt wirklich und abgeschlossen ist und zwar zu einer bestimmten, anderweitig festgelegten Zeit und dass er die Gesprächsbeteiligten unmittelbar angeht. Bei atelischen Verben hingegen bedeutet das Perfekt, dass ein Sachverhalt wirklich und $[\ldots]$ vergangen ist $[\ldots]$. (DpG: 638)

Das Plusquamperfekt wird als „,vorvergangen im Verhältnis zu einem anderen Sachverhalt oder als zu einem vergangenen Zeitpunkt abgeschlossen" definiert (DpG: 641). An dieser Stelle muss ich hervorheben, dass diese Definitionen den Default-Fall betreffen. Da zurzeit an einer Neuauflage der Grammatik gearbeitet wird, sind in der Neufassung viele Modifizierungen geplant, die einerseits kritische Stimmen zur DpG, den neueren Forschungsstand und andererseits im stärkeren Maße die Bedürfnisse der anvisierten Benutzer berücksichtigen.

\subsection{Die Duden-Grammatik}

In der Duden-Grammatik (2006) werden zunächst die Aktionsarten behandelt und telische, atelische sowie neutrale Verben unterschieden. Mit Recht stellt die Verfasserin des Kapitels (Fabricius-Hansen) fest:

Die Aktionsart eines Verbs ist in vielen Fällen nicht ein für alle Mal festgelegt, sondern eher als ein Potential aufzufassen, das je nach dem Zusammenhang, in dem das Verb erscheint, die eine oder die andere Richtung nehmen kann. (Duden: 416)

Im Weiteren wird an einigen Beispielen veranschaulicht, dass mit atelischen Verben auch die Außenperspektive (= Erreichung einer Grenze) im Präteritum vermittelt werden kann:

(14) Ich ging nach Hause, duschte, schlief und ging dann wieder aus.

${ }^{12}$ Da ich Verfasser des Kapitels „Das Verb“ in der DpG bin, muss ich meine Kritik an manchen Lösungen beschränken. 
Die Verfasserin weist darauf hin, dass bei den Perfekttempora das Geschehen ,als vorzeitig oder abgeschlossen mit Bezug auf die Orientierungszeit dargestellt" (Duden: 505) wird: „Abhängig von der Aktionsart des Prädikats und dem allgemeinen Zusammenhang erlaubt das Präteritum eine Außenperspektive oder eine Binnenperspektive auf das Geschehen“ (Duden: 518).

Bei der Beschreibung des Präsens- und Präteritumperfekts jedoch steht das Merkmal ,vollzogen“ in runden Klammern, was die Deutung erschwert:

Das Präsensperfekt dient als Vorzeitigkeitstempus im Verhältnis zum Präsens. Das bedeutet: Das Vollverbgeschehen wird als vorzeitig (vollzogen) präsentiert relativ zu einem Orientierungszeitpunkt, der in Übereinstimmung mit der Funktion des einfachen Präsens festgelegt ist. (Duden: 513)

\title{
4. Zusammenfassende Bemerkungen
}

Einige beachtenswerte Hinweise zum Perfekt im Sinne der Komplexitätshypothese finden wir u. a. bei Ehrich/Vater (1989: 109f.):

Obwohl die Grundbedeutung des Perfekts stets dieselbe ist, wird man das Perfekt von Verben unterschiedlicher Aktionsart unterschiedlich deuten: bei resultativen Verben (accomplishments und achievements in der Terminologie Vendlers) als Präsensperfekt, bei durativ-nicht-resultativen Verben (activities und states) als unbestimmte Vergangenheit und bei nichtdurativ-nichtresultativen Verben (semelfactives) als Perfekt der unmittelbaren Vergangenheit.

Wichtige und an zahlreichen Beispielen veranschaulichte Beobachtungen zur aktionalen Charakteristik ${ }^{13}$ der Vergangenheitstempora enthält die klassische Arbeit von Thieroff (1982: 188):

\begin{abstract}
Wie für das Präteritum gilt auch für das Perfekt, daß Abgeschlossenheit zum Sprechzeitpunkt nur bei punktuellen und telischen Verben zwingend gegeben ist, daß also die Abgeschlossenheit in Karl ist vom Stuhl gefallen (das nicht mit und er fällt immer noch fortgeführt werden kann) allein auf die Situationsklasse des Verbs, nicht auf die Tempusform zurückzuführen ist.
\end{abstract}

Die aktionale Charakteristik der deutschen Verben hat Andersson $(1972 ; 1978)$ am ausführlichsten dargestellt. Seine Einteilung der Verben in drei Gruppen, und zwar in telische, altelische und bedingt telische (neutrale), sowie die Untersuchung, welchen Einfluss diese Aktionsarten auf die Erreichung/Nichterreichung einer Grenze der Handlung (EG/NEG) ausüben, hat die Forschung zur Aktionalität im Deutschen stark beeinflusst. Die Opposition EG/NEG „,befindet sich auf der kontextuellen Satz- (oder satzverbindenden) Ebene“ (Andersson 1972: 185); beide Begriffe beinhalten die se-

${ }^{13}$ Thieroff (1982: 25-33) unterscheidet drei Klassen von Situationen: atelische, telische und punktuelle Situationen. 
mantische Entsprechung für die Aspektopposition in den slawischen Sprachen: „Der perfektive Aspekt [drückt] die Erreichung und Überwindung einer Grenze der Handlung aus, während der imperfektive dies nicht tut" (Andersson 1972: 62). Die Analyse von Übersetzungen aus dem Russischen ins Deutsche erlaubte es dem Verfasser, eine Reihe von Äquivalenten für den russischen Aspekt im Deutschen zu finden und zu beschreiben. Die Wahl der grammatischen und lexikalischen Mittel zum Ausdruck der EG/NEG ist im Deutschen fakultativ, so dass die Feststellung, ob EG oder NEG ausgedrückt wird, nicht immer eindeutig festzustellen sei. Neben der aktionalen Charakteristik des Verbs sind die temporalen und lokalen Adverbialien, temporale Konjunktionen, Präpositionalphrasen, Numerus und Determiniertheit/Nicht-Determiniertheit des Objekts, Funktionsverbgefüge, Tempora und Passiv sowie Kohärenzschemata (z. B. Parallelität, Aufeinanderfolge, Eintritt der Handlung) zu berücksichtigen. Die Analysen von Andersson liefern wichtige Hinweise zum Einfluss der aktionalen Charakteristik der Verben und des Kontextes auf die deutschen Tempora.

Die aktionalen Merkmale des Verbs (der Verbalphrase) telisch, atelisch spielen mit einigen grammatischen Kategorien und Erscheinungen des Deutschen zusammen; hier nur einige Beispiele:

- Bei dem Präteritum, Perfekt und Plusquamperfekt bewirkt das telische Verb beim geeigneten Kontext den Vollzug der Handlung, bei den atelischen - nur die Vergangenheit.

- Beim Perfekt und Futur II zur Bezeichnung eines zukünftigen Geschehens ist nicht nur die temporale zukunftsbezogene Angabe, sondern auch ein telisches (resultatives) Verb notwendig - somit weisen diese Varianten die Bedeutung ,abgeschlossen" auf.

- Die Unterscheidung zwischen den beiden Varianten von Futur I hängt mit der aktionalen Charakteristik des Infinitivs zusammen: Bei den Zustandsverben (d. h. prototypisch atelischen Verben) liegt hauptsächlich die Variante „Gegenwartsbezug mit Vermutung“vor. Bei telischen Verben ist in der Regel mit dem Zukunftsbezug zu rechnen (man muss hier jedoch anmerken, dass die Grenzen zwischen den telischen und atelischen Verben/Verbphrasen fließend sind, da die endgültige Deutung von vielen Faktoren abhängig ist):

(15) Er wird in die Schule gehen. (tel.)

Pójdzie do szkoły. (perf. Futur)

(16) Er wird noch in die Schule gehen. (atel., iter.)

Chodzi chyba jeszcze do szkoły. (imperf., iter.)

(17) Sie wird krank sein. (atel.) Jest chyba chora. (Präsens, Gegenwart)

- Bei den Modalverben legt der atelische Infinitiv I die epistemische, der telische dagegen - die deontische Deutung nahe; auf diese Regel hat Werner Abraham in mehreren Beiträgen hingewiesen:

(18) Er muss krank, böse sein. (epistemisch, Vermutung)

(19) Er muss den Bericht abgeben. (deontisch, Notwendigkeit) 
Bei der Deutung des Satzes mit den Modalverben muss man außerdem die Textsorte, die Person und die Zeitreferenz berücksichtigen.

Ich hoffe, dass meine Ausführungen gezeigt haben, dass bei der Beschreibung von Tempora die Berücksichtigung der aktionalen Charakteristik des Verbs unumgänglich ist, insbesondere in der Grammatik für den Ausländerunterricht (oder in der kontrastiven Grammatik); aus Platzgründen konnte ich nicht auf die Textfunktionen der deutschen Tempora eingehen - hier kann ich u. a. auf die Studien von Marschall (1995), Gelhaus (1974) sowie Hennig (2000) verweisen.

\section{Literatur}

Abraham, Werner/Meihsner, Peter/Piskorz, Jadwiga/Piskorz, Kinga (2011): „Zur paradigmatischen Ausbildung von Modalverben, von Deontik und Epistemik im jüngeren gesprochenen Polnisch.“ In: Kątny, Andrzej/Lukas, Katarzyna (Hgg.): Germanistik in Polen. Geschichte, Perspektiven, interdisziplinärer Dialog. Frankfurt a. M. u. a.: Peter Lang Verlag. 157-190.

Andersson, Sven-Gunnar ([1972] 1978): Aktionalität im Deutschen. Eine Untersuchung unter Vergleich mit dem russischen Aspekt. Bd. I., Bd. II (1978). Uppsala: Almqvist \& Wiksell.

Andersson, Sven-Gunnar (2004): „Gibt es Aspekt im Deutschen?““ In: Gautier, Laurent/Haberkorn, Didier (Hgg.): Aspekt und Aktionsart im heutigen Deutsch. Tübingen: Stauffenburg. 1-11.

Baumgärtner, Klaus/Wunderlich, Dieter (1969): „Ansatz zu einer Semantik des deutschen Tempussystems.“ In: Der Begriff Tempus - eine Ansichtssache? Düsseldorf: Schwann. 5-22.

Brinkmann, Hennig (1971): Die deutsche Sprache. Gestalt und Leistung. 2., neubearbeitete und erweiterte Auflage. Düsseldorf: Schwann.

Bull, William ( $\left.{ }^{3} 1968\right)$ : Time, Tense, and the Verb. Berkeley/Los Angeles: University of California Press.

Comrie, Bernard (1985): Tense. Cambridge: Cambridge University Press.

Czochralski, Jan (1975): Verbalaspekt und Tempussystem im Deutschen und Polnischen. Eine konfrontative Darstellung. Warszawa: Państwowe Wydawnictwo Naukowe.

Darski, Józef (1999): Bildung der Formen im Standarddeutschen. Tübingen: Stauffenburg.

Darski, Józef (2004): „Die deutsche Grammatik in Theorie und Didaktik.“ In: Dębski, Antoni/Lipiński, Krzysztof (Hgg.): Perspektiven der polnischen Germanistik in Sprach- und Literaturwissenschaft. Kraków: Wydawnictwo UJ. 299-311.

Darski, Józef (2015): Deutsche Grammatik. Ein völlig neuer Ansatz. 2., aktualisierte und erweiterte Auflage. Frankfurt a. M. u. a.: Peter Lang Verlag.

DpG (1999): Engel, Ulrich u. a.: Deutsch-polnische kontrastive Grammatik. Bd. 1. Heidelberg: Groos.

Duden (2006): Die Grammatik. Bd. 4. 7., völlig neu erarbeitete und erweiterte Auflage. Mannheim: Dudenverlag.

Ehrich, Veronika (1992): Hier und jetzt. Studien zur lokalen und temporalen Deixis im Deutschen. Tübingen: Max Niemeyer Verlag.

Ehrich, Veronika/Vater, Heinz (1989): „Das Perfekt im Dänischen und Deutschen.“ In: Abraham, Werner/ Janssen, Theo (Hgg.): Tempus, Aspekt, Modus. Tübingen: Max Niemeyer Verlag. 103-132.

Engel, Ulrich (1988): Deutsche Grammatik. Heidelberg: Groos.

Engel, Ulrich (2004): Deutsche Grammatik. Neubearbeitung. München: Iudicium.

Eisenberg, Peter (1986): Grundriß der deutschen Grammatik. Stuttgart: Metzler.

Eisenberg, Peter (1999): Grundriß der deutschen Grammatik. Bd. 2: Der Satz. Stuttgart/Weimar: Metzler. Erben, Johannes ( $\left.{ }^{6} 1963\right)$ : Abriß der deutschen Grammatik. Berlin: Akademie-Verlag. 
Gelhaus, Hermann (1974): „Perfekt und Präteritum in Ein-Satz-Äußerungen der geschriebenen deutschen Sprache.“ In: Gelhaus, Hermann/Latzel, Sigbert (Hgg.): Studien zum Tempusgebrauch im Deutschen. Mannheim: IdS. 173-274.

Gross, Harro (1974): Der Ausdruck des Verbalaspekts in der deutschen Gegenwartssprache. Hamburg: Buske.

Heidolph, Karl u. a. (1981): Grundzüge einer deutschen Grammatik. Berlin: Akademie-Verlag.

Helbig, Gerhard/Buscha Joachim (2001; $\left.{ }^{1} 1972\right)$ : Deutsche Grammatik. Ein Handbuch für den Ausländerunterricht. Berlin: Langenscheidt.

Hennig, Mathilde (2000): Tempus und Temporalität in geschriebenen und gesprochenen Texten. Tübingen: Max Niemeyer Verlag.

Hentschel, Elke/Weydt, Harald (1990): Handbuch der deutschen Grammatik. Berlin: WdeG.

Jung, Walter ( $\left.{ }^{6} 1980\right)$ : Grammatik der deutschen Sprache. Neuausgabe, bearbeitet von Günter Starke. Leipzig: Bibliographisches Institut.

Kątny, Andrzej (2010): „Zu Resultativ und Modalverben in epistemischer Lesart aus kontrastiver Sicht.“ In: Kątny, Andrzej/Socka, Anna (Hgg.): Modalität / Temporalität in kontrastiver und typologischer Sicht. Frankfurt a. M. u. a.: Peter Lang Verlag. 59-75.

Kątny, Andrzej (2011): „Zum possessiven Resultativ in ausgewählten slawischen Sprachen.“ In: Kotin, Michail L./Kotorova, Elizaveta G. (Hgg.): Geschichte und Typologie der Sprachsysteme. Heidelberg: Winter. 181-188.

Leiss, Elisabeth (1992): Die Verbalkategorien des Deutschen. Berlin/New York: Walter de Gruyter.

Marschall, Matthias (1995): Textfunktionen der deutschen Tempora. Genève: Editions Slatkine.

Paul, Hermann ( $\left.{ }^{5} 1959\right):$ Deutsche Grammatik. Bd. IV: Syntax (zweite Hälfte). Halle (Saale): Max Niemeyer Verlag.

Piskorz, Jadwiga (2012): Die Grammatikalisierung eines neuen Perfekts im Polnischen. München: Otto Sagner.

Schmidt, Wilhelm (1977): Grundfragen der deutschen Grammatik. Berlin: Volk und Wissen.

Socka, Anna (1996): „Die Vergangenheitstempora im Deutschen und ihre polnischen Äquivalente.“ Convivium, $57-81$.

Thieroff, Rolf (1992): Das finite Verb im Deutschen. Tempus - Modus - Distanz. Tübingen: Gunter Narr Verlag.

Vater, Heinz (2002): „Präteritum und Perfekt im Deutschen“. Estudios Filológicos Alemanes 1, 35-72; auch in: Vater, Heinz (2010): Linguistik und deutsche Grammatik im Fokus. Ausgewählte Schriften; hrsg. von Andrzej Kątny. Gdańsk: Wydawnictwo UG. 347-377.

Vater, Heinz (2007): Einführung in die Zeit-Linguistik. 4., verb. und erw. Auflage. Trier: Wissenschaftlicher Verlag.

Žerebkov, Vadim (1975): Das Verb. Ein Hilfsbuch zur deutschen Grammatik. Moskau: Vysšaja škola. 